\title{
VALIDATION OF CFD FOR THE DETERMINATION OF DAMPING COEFFICIENTS FOR THE USE OF WAVE ENERGY CONVERTERS MODELLING
}

\author{
Olivia Thilleul \\ HydrOcean \\ Nantes, France
Aurélien Drouet
HydrOcean
Nantes, France

\author{
Aurélien Babarit \\ LUNAM Université \\ Ecole Centrale de Nantes - CNRS \\ Nantes, France
}

\begin{abstract}
Diffraction-radiation codes enable to model the behaviour of Wave Energy Converters (WEC) and seakeeping of ships on many sea-states with very little computational time. However, the viscous effects are neglected and therefore the simulations lead to relatively inaccurate values. The inaccuracy mainly occurs at the resonance frequency, especially in roll motions for which viscous effects are of major importance. Classically, the viscous effects are represented by adding viscous damping coefficients obtained either from experimental data or analytical approaches based on numerous approximations.
\end{abstract}

In order to improve the accuracy of the diffraction-radiation solvers, the damping coefficients can also be calculated from Computational Fluid Dynamics (CFD) simulations. The first part of this paper presents the three CFD solvers and turbulence models used in this validation study: ICARE and ISIS-CFD are developed by Ecole Centrale de Nantes and Star-CCM+ is a general purpose solver developed by $\mathrm{CD}$-adapco. For each case, a preferred solver is chosen and a second solver is used for verification in most cases.

The second part briefly presents the theory that obtains drag coefficients in oscillatory flows, which are closely related to damping coefficients in waves. Each of the three following parts introduces the experimental test cases to which numerical results are compared to. The numerical parameter convergence

\author{
Sébastien Le Floch \\ HydrOcean \\ Nantes, France
}

study leads to a choice of around 200 timesteps per period with an adapted mesh enabling to obtain drag coefficients with errors lower than 5\%. A mesh convergence study in the wake area leads to a mesh refinement of around 2 to $2.5 \%$ of the body characteristic length. In order to reduce the computational time, the total number of cells can be decreased by mainly refining locations where specific flow detachment occurs, such as body corners or sharp edges. Turbulence models are also varied. Validation results are finally presented in terms of single or coupled damping coefficients and added mass coefficients. They are presented for various non-dimensional numbers such as Keulegan-Carpenters and Reynolds number.

KEY WORDS: seakeeping, CFD, viscous damping coefficients, validation, Wave Energy Converters (WEC)

\section{INTRODUCTION}

Damping coefficients are necessary to obtain representative results with diffraction radiation codes. They are classically determined from free decay or forced oscillation motions in tank tests. They can also be computed from CFD computations. Numerous references in the literature are referring to model damping either experimentally or numerically.

Tao and Thiagarajan (2003) had shown that hydrodynamic damping exerted on an oscillating cylinder and disk is related to 
the vortex shedding modes. Bonfiglio (2012) performed CFD computations with OpenFoam solver and shows on overestimation of roll damping coefficients compared to experiments (Vugts, 1968). Atluri (2009) used CFD to compute hydrodynamic coefficients of oscillating bodies for offshore structured and validated the method on a flat plate. These references are non-exhaustive examples of comparable studies available in the literature.

The objective of this paper is to perform comparisons of damping coefficients between CFD simulations and experiments on various classical shapes. The various numerical parameters to obtain accurate results are determined and presented in this paper.

\section{NOMENCLATURE}

$$
\begin{aligned}
& \mathrm{K}_{c}: \text { Keulegan-Carpenter number } K_{c}=\frac{2 \pi A}{D} \\
& R_{e}: \text { Reynolds numer } R_{e}=\frac{U D}{v} \\
& \beta \text { : Stokes parameter with } \beta=\frac{R}{K_{c}}
\end{aligned}
$$

With $A$ : amplitude of oscillations

$U$ : velocity of oscillations

$D$ : body diameter or characteristic length

$v$ : kinematic viscosity if the fluid

\section{PRESENTATION OF SOLVERS AND TURBULENCE MODELS}

\section{Finite difference method with free surface tracking}

One of the solvers used in this paper is ICARE (Alessandrini, Delhommeau, 1994 and 1999), a free-surface RANS solver developed by the Ecole Centrale Nantes. The convective form of Reynolds Averaged Navier-Stokes Equations is written through partial transformation from Cartesian space $\left(x^{1}, x^{2}, x^{3}\right)$ to curvilinear space $\left(\xi^{1}, \xi^{2}, \xi^{3}\right)$ fitted to the hull and the free surface at each time step. The dependent unknowns of the system are the free surface elevation $(h)$, the three Cartesian velocity components $\left(u^{i}\right)$, the pressure $(p)$ including the gravitational effects $\left(\rho g x^{3}\right)$ and the turbulent kinetic energy $\left(\frac{2}{3} \rho k\right)$. Mean momentum transport equations are written in the moving reference frame attached to the hull. Mass conservation is expressed as the classical continuity equation. To close the equations set we use a classical $k-\omega$ turbulence model proposed by Wilcox (Wilcox, 1988), introducing a specific dissipation rate $\varpi$ without low Reynolds formulation requirement.

Free surface boundary conditions are the kinematic condition, the two tangential dynamic conditions and the normal dynamic condition. The kinematic condition comes from the continuity hypothesis that expresses that the fluid particles of free surface stay on it. Dynamic conditions of the free surface are given by the continuity of strains at the free surface.

General schemes are based on second order (in space and time) implicit finite differences. Discrete unknowns are distributed on a structured curvilinear grid fitted to the hull and the free surface. Velocity Cartesian components, kinetic turbulent energy and specific dissipation rates are located on the grid nodes. Pressure is located at the center of each volume and free surface elevation is located on the center of the free surface interfaces.

\section{Finite volume method and Volume Of Fluid (VOF) free surface}

Two solvers used in this study are based on a finite volume scheme with a VOF treatment of the free surface. One of them is ISIS-CFD, which was also developed by the Ecole Centrale de Nantes (Visonneau, 2012). The second one is Star-CCM+, which is a general-purpose code, developed by CD-adapco. They both resolve the incompressible Unsteady ReynoldsAveraged Navier Stokes equations in a bi-fluid formulation with a general face-based finite volume formulation. Freesurface water flows are modelled with a convection equation for the volume fraction of water $\alpha$. The flow equations are discretised in a finite-volume framework. Pressure-velocity coupling is obtained through a Rhie \& Chow SIMPLE-type method: in each time step, the velocity updates come from the momentum equations and the pressure is given by the mass conservation law, transformed into a pressure equation. As the volume fraction $\alpha$ is discontinuous, its convection equation is discretised with compressive flux functions.

The resolution of this equation in each time step is decoupled from the pressure and velocity updates. The discretisation is face-based. While all unknown state variables are cell-centred, the system of equations used in the implicit time stepping procedure are constructed face by face. Fluxes are computed in a loop over the faces and the contribution of each face is then added to the two cells next to the face. This technique requires no specific cell topology. Therefore, the grids can be completely unstructured. Cells with an arbitrary number of arbitrarily shaped faces are accepted.

Several turbulence models ranging from one-equation model to Reynolds stress transport model are implemented. The one equation Spalart-Allmaras model is a model with integration to wall based on a transport equation for the turbulent viscosity. This model does not provide good prediction in jet flows, but gives reasonably good predictions of two-dimensional mixing layers, wake flows, and flat-plane boundary layers and shows improvements in the prediction of flows with adverse pressure gradients compared with the $K-\varepsilon$ and $K-\omega$ model, although not as much as the SST $K-$ model. 
The SST $K-\omega$ (shear-stress transport) model combines several desirable elements of existing two-equation models. The two major features of this model are a zonal blending of model coefficients and a limitation of the growth of the eddy viscosity in rapidly strained flows. Two-equation models generally under-predict the retardation and separation of the boundary layer due to adverse pressure gradients. The reason of this deficiency is that two-equation models do not account for the important effects of transport of the turbulent stresses. However, three-dimensional calculations on other flows do not seem to always confirm a superiority of the SST version with respect to the baseline form.

The $K-\varepsilon$ turbulence model is a two-equation model in which transport equations are solved for the turbulent kinetic energy $K$ and its dissipation rate $\varepsilon$. The $K-\varepsilon$ turbulence model has been shown to be useful for free-shear layer flows with relatively small pressure gradients.

\begin{tabular}{|c|c|c|c|}
\hline & $\begin{array}{l}\text { Numerical } \\
\text { scheme }\end{array}$ & Mesh & $\begin{array}{c}\text { Free } \\
\text { surface }\end{array}$ \\
\hline ICARE & $\begin{array}{c}\text { Finite } \\
\text { difference }\end{array}$ & Structured & $\begin{array}{l}\text { Tracking } \\
\text { method }\end{array}$ \\
\hline ISIS-CFD & Finite volume & Unstructured & VOF \\
\hline Star-CCM+ & Finite volume & Unstructured & VOF \\
\hline
\end{tabular}

Table 1: General characteristics of the CFD solvers used in the validation study

\section{VALIDATIONS OF CFD DAMPING COEFFICIENT CALCULATIONS}

\section{Obtaining damping coefficients from forced oscillation motions}

In this paper, the calculation of damping coefficients is based on forced oscillation motions. The force or moment acting on the body is identified either to the Morison formulation or to the theoretical expression of forces. It enables to obtain either a drag coefficient $C_{d}$ in oscillatory flow, in phase with the velocity, or the linear and quadratic damping coefficients in phase with the velocity and the square velocity. The imposed motion is written $\varphi(t)=\varphi_{0} \cos (\omega t)$

The first assumption is that the signal of forces or moments is based on a harmonic decomposition at the first order:

$$
F(t)=a_{1} \cos (\omega t)+b_{1} \sin (\omega t)
$$

The force or moment at first order is identified to the general Morison formulation:

$$
F(t)=\rho S\left(C_{M} \ddot{X}-\left(C_{M}-1\right) \ddot{\varphi}\right)+\frac{1}{2} \rho C_{D} D(\dot{X}-\dot{\varphi})|\dot{X}-\dot{\varphi}|
$$

With $\dot{X}$ : the flow velocity and $\dot{\varphi}$ the body velocity. In this paper, the flow velocity $\dot{X}=0$ and the body is in forced motions, which leads to:

$$
F(t)=\rho S\left(1-C_{M}\right) \ddot{\varphi} \quad \frac{1}{2} \rho C_{D} D \dot{\varphi}|\dot{\varphi}|
$$

It then leads to:

$$
\begin{gathered}
C_{M}=1+\frac{a_{1}}{\rho S \omega^{2} \varphi_{0}} \\
C_{D}=\frac{b_{1}}{\frac{1}{2} \rho D \frac{8}{3 \pi}{ }^{2} \varphi_{0}^{2}}
\end{gathered}
$$

\section{SQUARE CYLINDER IN OSCILLATORY FLOW}

\section{Experimental set up}

These experiments were performed by Bearman et al (1985) in a U-tube. The tested geometry is a cylinder with a square section of $2.67 \mathrm{~cm}$ side length and a length of $60.5 \mathrm{~cm}$. The sine oscillations of the fluid in the U-tube are performed for a period of $3.34 \mathrm{~s}$. Cases are performed for $\mathrm{K}_{\mathrm{c}}$ numbers and corresponding Reynolds numbers presented in Table 7.

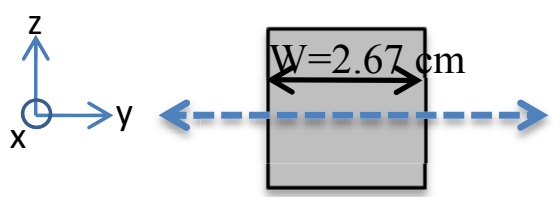

Figure 1: Sketch of the square section studied in experiments from Bearman (1985)

\begin{tabular}{|c|c|c|c|c|c|c|c|c|}
\hline $\mathbf{K}_{\mathbf{C}}$ & $\mathbf{1 . 1 2}$ & $\mathbf{1 . 3 0}$ & $\mathbf{1 . 4 9}$ & $\mathbf{1 . 6 5}$ & $\mathbf{1 . 8 7}$ & $\mathbf{2 . 2 0}$ & $\mathbf{2 . 6 7}$ & $\mathbf{3 . 1 1}$ \\
\hline $\mathbf{R}_{\mathbf{e}}$ & 239 & 277 & 318 & 352 & 398 & 470 & 571 & 664 \\
\hline
\end{tabular}

\begin{tabular}{|c|c|c|c|c|c|c|c|c|}
\hline $\mathbf{K}_{\mathbf{C}}$ & $\mathbf{3 . 5 5}$ & $\mathbf{4 . 1 3}$ & $\mathbf{4 . 5 6}$ & $\mathbf{4 . 8 4}$ & $\mathbf{5 . 5 0}$ & $\mathbf{7 . 0 5}$ & $\mathbf{8 . 2 9}$ & $\mathbf{9 . 4 2}$ \\
\hline $\mathbf{R}_{\mathbf{e}}$ & 757 & 881 & 974 & 1033 & 1174 & 1505 & 1770 & 2012 \\
\hline
\end{tabular}

Table 2: Definition of $\mathrm{Kc}$ and corresponding $\mathrm{Re}$

Numerical model

The numerical model is performed as a $2 \mathrm{D}$ case. Two references are used: a fixed reference frame is used for the flow and the mesh is setup with a moving reference frame to enable the oscillation of the body.

\section{Mesh convergence}

The mesh convergence study on the square section is performed for $\mathrm{Kc}=2.5$ on four different mesh densities presented in Figure 2 and Table 3. All meshes are built in order to have a $\mathrm{y}+$ lower than 1 to enable a direct resolution in the boundary layer. The timestep is adapted to have a CFL 
condition equal to one. The turbulence model is SST $\mathrm{K}-\omega$. The mesh $\mathrm{M}_{2}$ is refined in corners, enabling to divide by two the total number of cells compared to the mesh $M_{1}$ with a negligible influence on the result's quality as shown in Figure 3. The mesh $M_{3}$ is similar to $M_{2}$ except that the length of the refined area in the wake is equal to twelve times the square section width (W) instead of four times for mesh $\mathrm{M}_{2}$.
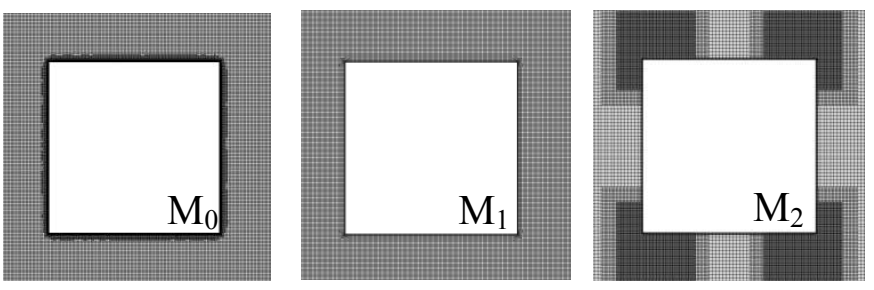

Figure 2: Views of the meshes $M_{0}, M_{1}, M_{2}$ on the square section

\begin{tabular}{|c|c|c|c|c|}
\hline & $\begin{array}{c}\text { Mesh size in } \\
\text { wake area in } \\
\% \text { of D }\end{array}$ & $\begin{array}{c}\text { Nb of } \\
\text { cells }\end{array}$ & $\begin{array}{c}\text { Nb of cells in } \\
\text { the boundary } \\
\text { layer }\end{array}$ & $\begin{array}{c}\text { B.Layer } \\
\text { thickness } \\
\text { in \% of D }\end{array}$ \\
\hline $\mathrm{M}_{0}$ & $1 \%$ & 107575 & 20 & $2.5 \%$ \\
\hline $\mathrm{M}_{1}$ & $1 \%$ & 93775 & 4 & $1.25 \%$ \\
\hline $\mathrm{M}_{2}$ & $\begin{array}{c}2 \% \\
\text { Wake: L=4W } \\
0.5 \% \text { (corners) }\end{array}$ & 56628 & 4 & $1.25 \%$ \\
\hline $\mathrm{M}_{3}$ & $\begin{array}{c}2 \% \\
\text { Wake: } \mathrm{L}= \\
12 \mathrm{~W} \\
0.5 \% \text { (corners) }\end{array}$ & 113368 & 4 & $1.25 \%$ \\
\hline
\end{tabular}

Table 3: Details of the four mesh densities for the square section

Figure 3 shows significant differences between computations and experiments at low $\mathrm{K}_{\mathrm{c}}$, which may be explained by the measurements difficulties in experiments to obtain the phase shift between the measured forces and the imposed motion.

Results are satisfactory for an intermediate $\mathrm{K}_{\mathrm{c}}$ (between 3 and 7), however they diverge at a high $\mathrm{K}_{\mathrm{c}}$ with the $\mathrm{M}_{2}$ mesh. This occurs for the highest motion amplitude. An analysis of the velocity fields (Figure 4) enabled to determine that the wake of the square section was alternatively in a coarse or a refined zone of the $\mathrm{M}_{2}$ mesh in the moving reference frame. Since the square passes through its wake during the oscillatory motion, this weak description of the wake at high amplitude motions decreases the result's quality. To improve the results, the $\mathrm{M}_{3}$ mesh is refined over a longer distance in the wake, providing satisfactory results at high $\mathrm{K}_{\mathrm{c}}$.

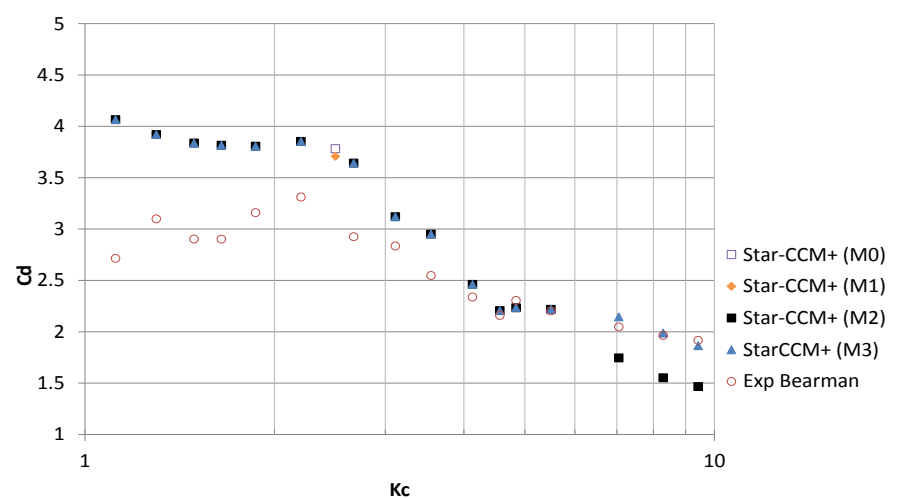

Figure 3: Mesh convergence study $_{4}$ on the square section

\begin{tabular}{|c|c|c|c|c|c|}
\cline { 2 - 6 } \multicolumn{1}{c|}{} & & $\mathrm{M} 0$ & $\mathrm{M} 1$ & $\mathrm{M} 2$ & $\mathrm{M} 3$ \\
\hline \multirow{2}{*}{$\mathrm{Kc}=2.5$} & $\mathrm{Cd}$ & $1.8 \%$ & $0.2 \%$ & $0.0 \%$ & REF. \\
\cline { 2 - 6 } & $\mathrm{Cm}$ & $1.3 \%$ & $0.1 \%$ & $0.0 \%$ & REF. \\
\hline \multirow{2}{*}{$\mathrm{Kc}=9.4$} & $\mathrm{Cd}$ & - & - & $27.2 \%$ & REF. \\
\cline { 2 - 6 } & $\mathrm{Cm}$ & - & - & $11.6 \%$ & REF. \\
\hline
\end{tabular}

Table 4: Differences in \% for the mesh convergence study on the square section

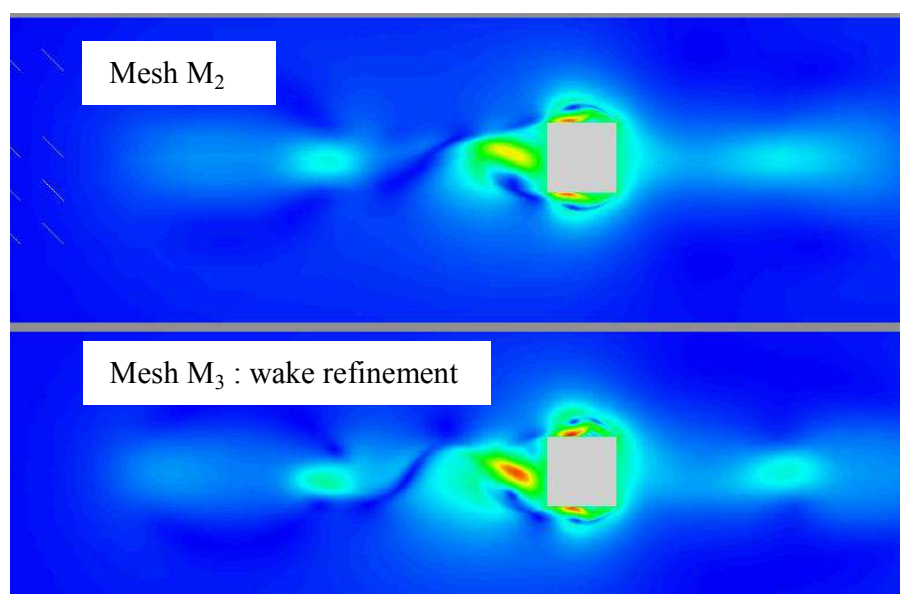

Figure 4: Difference on the velocity field between meshes $M_{2}$ and $\mathbf{M}_{3}$

\section{Timestep and nonlinear iterations study}

A timestep convergence study is performed on the square sections. It is performed for the $\mathrm{M}_{2}$ mesh. Table 5 shows that 250 timesteps per period of forced oscillations leads to errors of around $1 \%$ on the added mass and drag coefficients and is sufficient for these computations.

\begin{tabular}{|c|c|c|c|c|}
\hline & $100 \mathrm{dt} / \mathrm{T}$ & $250 \mathrm{dt} / \mathrm{T}$ & $500 \mathrm{dt} / \mathrm{t}$ & $1000 \mathrm{dt} / \mathrm{T}$ \\
\hline $\mathrm{Cd}$ & $4.54 \%$ & $1.29 \%$ & $0.39 \%$ & $\mathrm{REF}$. \\
\hline $\mathrm{Cm}$ & $1.98 \%$ & $0.57 \%$ & $0.17 \%$ & $\mathrm{REF}$. \\
\hline
\end{tabular}

Table 5 : Timestep convergence on the square section

\begin{tabular}{|c|c|c|c|c|}
\hline & 10 itnonlin & 20 itnonlin & 30 itnonlin & 100 itnonlin \\
\hline $\mathrm{Cd}$ & $4.73 \%$ & $1.33 \%$ & $0.53 \%$ & REF. \\
\hline $\mathrm{Cm}$ & $2.10 \%$ & $0.59 \%$ & $0.24 \%$ & REF. \\
\hline
\end{tabular}

Table 6: Nonlinear iterations study on the square section 


\section{Turbulence model influence}

Three different turbulence models are tested on the square section for $\mathrm{K}_{\mathrm{c}}=2.5$. The results presented in Table 7 do not show any significant differences between SST $K-\omega, K-\varepsilon$ and Spalart-Allmaras model.

\begin{tabular}{|c|c|c|c|}
\cline { 2 - 4 } \multicolumn{1}{c|}{} & Spalart-Allmaras & $\mathrm{k}-\varepsilon$ & $\mathrm{k}-\omega$ \\
\hline $\mathrm{Cd}$ & $4.6 \%$ & $2.2 \%$ & $\mathrm{REF}$. \\
\hline $\mathrm{Cm}$ & $0.5 \%$ & $2.1 \%$ & $\mathrm{REF}$. \\
\hline
\end{tabular}

Table 7: Influence of the turbulence model for the square section

\section{Validation results}

The results obtained after completing the influence of numerical parameters are presented in Figure 5 and Figure 6. They show correct results on the drag coefficient at high $\mathrm{K}_{\mathrm{c}}$ and some discrepancies are observed at low $\mathrm{K}_{\mathrm{c}}$. Various references had shown that using a turbulence model at low $\mathrm{K}_{\mathrm{c}}$ is not necessary to perform forced oscillation tests. Atluri et al. (2009) performed forced oscillation motions on various components of an offshore platform like heave plates. They obtained same results with and without turbulence at low $\mathrm{Re}$ and $\mathrm{K}_{\mathrm{c}}$, and deduced that modelling turbulence is not necessary. Kinnas et al. (2003) modelled a separated flow due to oscillations past a $2 \mathrm{D}$ vertical plate. The numerical model solving Euler and Navier-Stokes equations provided comparable results at low $\mathrm{K}_{\mathrm{c}}$. Hence, laminar computations should also be performed on the square section of Bearman since these cases are performed at low $\mathrm{K}_{\mathrm{c}}$ and low Re.

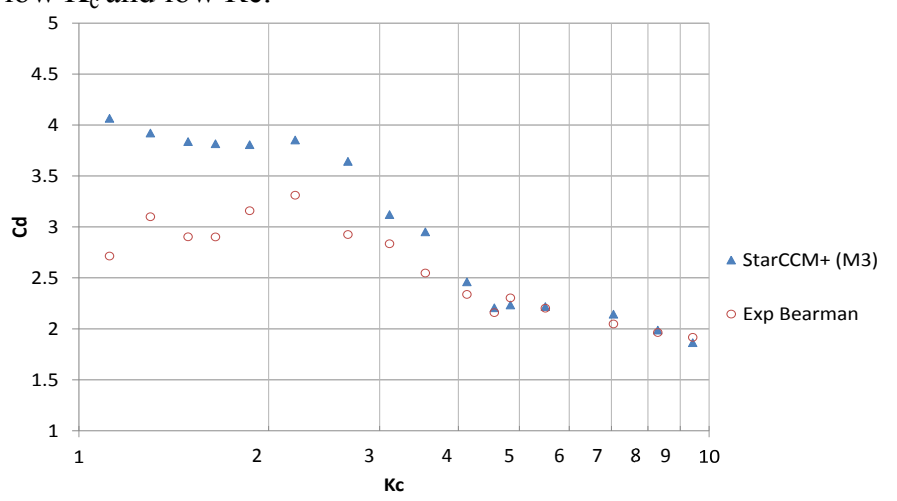

Figure 5: Validation for $C_{d}$ on the square section (SST

$\omega)$

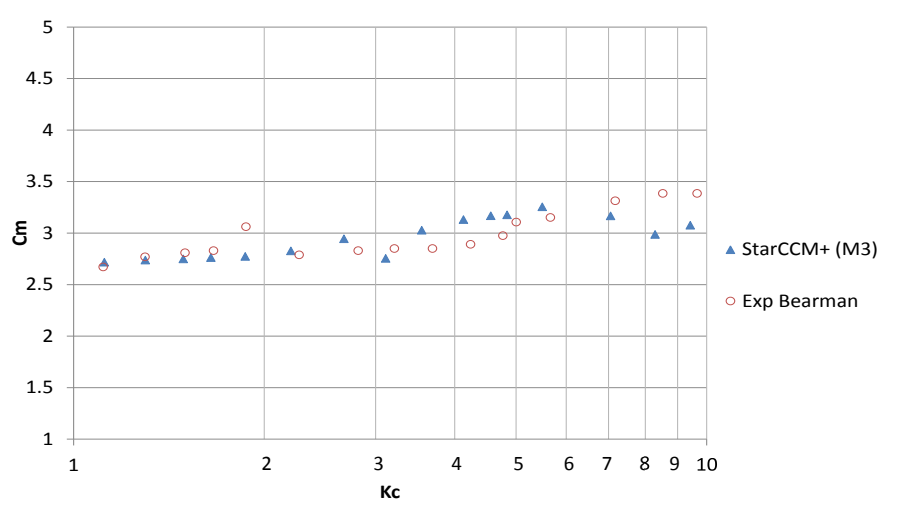

Figure 6: Validation for $\mathrm{C}_{\mathrm{m}}$ on the square section (SST

\section{CIRCULAR CYLINDER IN OSCILLATORY FLOW}

\section{Experimental set up}

Sarpkaya's experiments (1976) were performed in a Utube. Five cylinders with circular sections are tested, with diameters $(D)$ varying from $5.08 \mathrm{~cm}$ (and corresponds to a Stokes parameter $\beta=497)$ to $16.51 \mathrm{~cm}(\beta=5260)$. For each tested diameter, the experiments are carried out for a varying Keulegan-Carpenter number from 4 to 50 . The $\mathrm{K}_{\mathrm{c}}$ numbers for which numerical computations are performed are presented in Table 8 and Table 9 with the corresponding forced oscillation amplitude and Reynolds numbers.

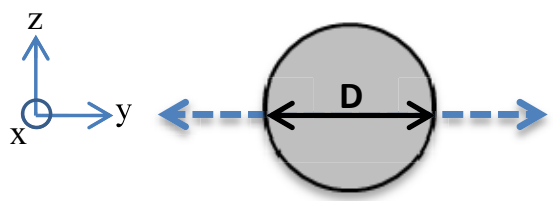

Figure 7: Sketch of the circular section studied in experiments from Sarpkaya (1976)

\begin{tabular}{|c|c|c|c|c|c|c|c|}
\hline $\begin{array}{c}\mathrm{A} \\
(\mathrm{m})\end{array}$ & 0.081 & 0.105 & 0.162 & 0.243 & 0.323 & 0.404 & \\
\hline $\mathrm{K}_{\mathrm{C}}$ & 10 & 13 & 20 & 30 & 40 & 50 & \multirow{2}{\sigma}{} \\
\cline { 1 - 6 } & $\begin{array}{c}4.97 .1 \\
\mathrm{R}_{\mathrm{e}}\end{array}$ & $\begin{array}{c}6.46 .1 \\
0^{3}\end{array}$ & $\begin{array}{c}9.94 .1 \\
0^{3}\end{array}$ & $\begin{array}{c}1.49 .1 \\
0^{4}\end{array}$ & $\begin{array}{c}1.99 .1 \\
0^{4}\end{array}$ & $\begin{array}{c}2.49 .1 \\
0^{4}\end{array}$ & $\curvearrowleft$ \\
\hline
\end{tabular}

Table 8: Kc and Re numbers of cases computed at $\beta=497$

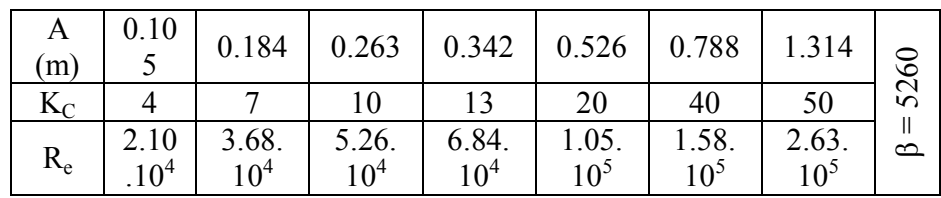

Table 9: Kc and $\operatorname{Re}$ numbers of cases computed at $\beta=5260$

\section{Numerical model}

In computations, the highest and lowest cylinder diameters are modeled as 2D cases. A fixed and a moving reference frame are used as for the square section case. The timestep and mesh convergence studies are performed on the $16.51 \mathrm{~cm}$ diameter and $\mathrm{K}_{\mathrm{c}}=4$ cylinder. The size of the domain is $6.6 \times 3.3 \mathrm{~m}$ and the mesh is refined in the wake area over a zone of $2 \times 1 \mathrm{~m}$. Five various mesh densities are studied with a $\mathrm{y}+$ lower than one and an aspect cell ratio close to the body equal to 1.2. Meshes are presented in Figure 8 and Table 10. The turbulence model is SST $K-$ 


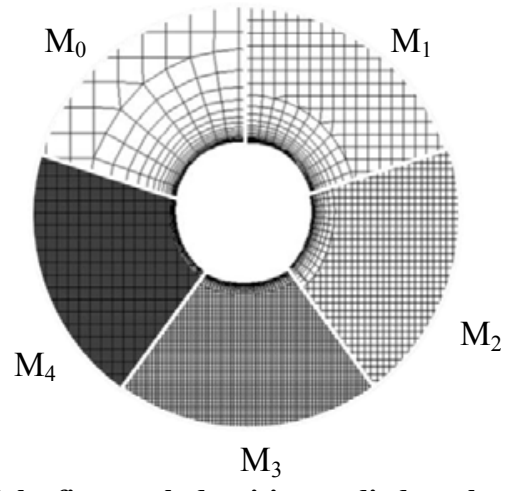

Figure 8: Views of the five mesh densities studied on the circular section

\begin{tabular}{|c|c|c|c|c|}
\hline & $\begin{array}{c}\text { Mesh size in } \\
\% \text { of } \mathrm{D}\end{array}$ & Nb of cells & $\begin{array}{c}\text { Nb of cells in the } \\
\text { boundary layer }\end{array}$ & $\begin{array}{c}\text { BL } \\
\text { thickness in } \\
\% \text { of } \mathrm{D}\end{array}$ \\
\hline $\mathrm{M}_{0}$ & $20 \%$ & 6624 & 26 & $69 \%$ \\
\hline $\mathrm{M}_{1}$ & $10 \%$ & 15070 & 24 & $35 \%$ \\
\hline $\mathrm{M}_{2}$ & $5 \%$ & 38781 & 21 & $17.2 \%$ \\
\hline $\mathrm{M}_{3}$ & $2.5 \%$ & 121564 & 12 & $8.36 \%$ \\
\hline $\mathrm{M}_{4}$ & $1.25 \%$ & 418012 & 16 & $4.3 \%$ \\
\hline
\end{tabular}

Table 10: Details of the five mesh densities for the circular section

Results presented in Table 11 show that correct results are obtained for the meshes $\mathrm{M}_{2}$ and $\mathrm{M}_{3}$ around $1.2 \times 10^{5}$ cells and 200 timesteps per period.

\begin{tabular}{|c|c|c|c|c|c|}
\hline $\mathrm{Cm}$ & $\mathrm{T} / \mathrm{dt}=25$ & $\mathrm{~T} / \mathrm{dt}=50$ & $\mathrm{~T} / \mathrm{dt}=100$ & $\mathrm{~T} / \mathrm{dt}=200$ & $\mathrm{~T} / \mathrm{dt}=400$ \\
\hline $\mathrm{M} 0$ & $3.4 \%$ & $2.7 \%$ & $2.7 \%$ & & \\
\hline $\mathrm{M} 1$ & $2.8 \%$ & $1.2 \%$ & $0.7 \%$ & $0.2 \%$ & \\
\hline $\mathrm{M} 2$ & $2.1 \%$ & $0.0 \%$ & $0.4 \%$ & $1.4 \%$ & $0.3 \%$ \\
\hline $\mathrm{M} 3$ & & $0.8 \%$ & $2.0 \%$ & $0.7 \%$ & $0.2 \%$ \\
\hline $\mathrm{M} 4$ & & & $1.0 \%$ & $0.7 \%$ & $\mathrm{REF}$. \\
\hline
\end{tabular}

\begin{tabular}{|c|c|c|c|c|c|}
\hline $\mathrm{Cd}$ & $\mathrm{T} / \mathrm{dt}=25$ & $\mathrm{~T} / \mathrm{dt}=50$ & $\mathrm{~T} / \mathrm{dt}=100$ & $\mathrm{~T} / \mathrm{dt}=200$ & $\mathrm{~T} / \mathrm{dt}=400$ \\
\hline $\mathrm{M} 0$ & $2.9 \%$ & $4.0 \%$ & $6.4 \%$ & & \\
\hline $\mathrm{M} 1$ & $6.8 \%$ & $3.4 \%$ & $2.0 \%$ & $2.5 \%$ & \\
\hline $\mathrm{M} 2$ & $9.6 \%$ & $6.2 \%$ & $5.5 \%$ & $6.2 \%$ & $2.0 \%$ \\
\hline $\mathrm{M} 3$ & & $6.6 \%$ & $6.2 \%$ & $1.1 \%$ & $3.1 \%$ \\
\hline $\mathrm{M} 4$ & & & $4.7 \%$ & $1.4 \%$ & REF. \\
\hline
\end{tabular}

Table 11 : Timestep and mesh convergence study on the circular section for added mass (above) and drag coefficient (below)

\section{Turbulence model influence}

The circular section is studied with both SST $K-\omega$ and $K \quad$ models. Results presented in Figure 9 show that the SST $K \quad$ turbulence model provides correct results for $\beta=497$ and $\beta=5260$ at low $\mathrm{K}_{\mathrm{c}}$. At high $\mathrm{K}_{\mathrm{c}}$, the $K-\varepsilon$ model provides better results. More generally, the results show significant differences depending on the choice of the turbulence model. However, the case of the circular section is more difficult to model since the flow detachment is not encouraged by sharp edges.

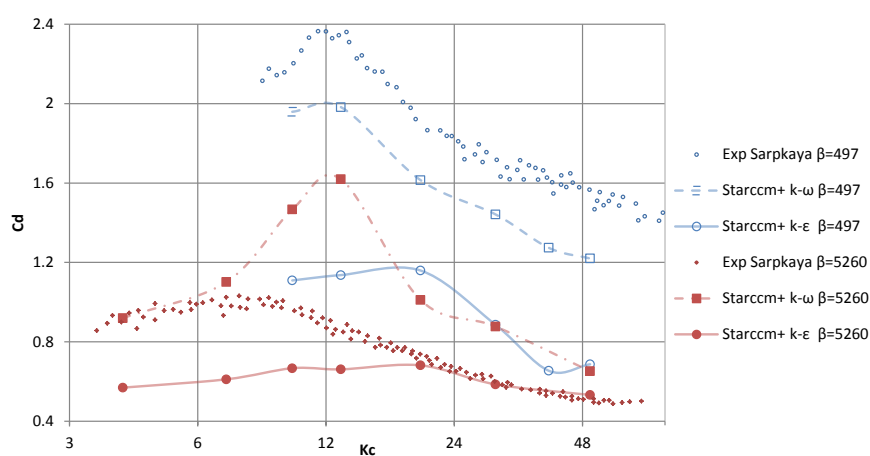

Figure 9: Influence of the turbulence model on the drag coefficient for the circular section

Figure 10 to Figure 12 show the velocity and vorticity fields at the maximum speed time instant of the forced motion for the two turbulence models. The SST $K-\omega$ model shows important and unexpected flow detachment at low and intermediate $\mathrm{K}_{\mathrm{c}}$. Indeed, the alternative vortex shedding is more visible with the $K-\varepsilon$ model for all $\mathrm{K}_{\mathrm{c}}$ numbers. At high $\mathrm{Kc}$, the velocity and vorticity fields are comparable with the two turbulence models. However, since drag coefficients calculated with the SST $K-\omega$ model provide results in better agreement with experiments, the following part of the study is performed with this turbulence model.
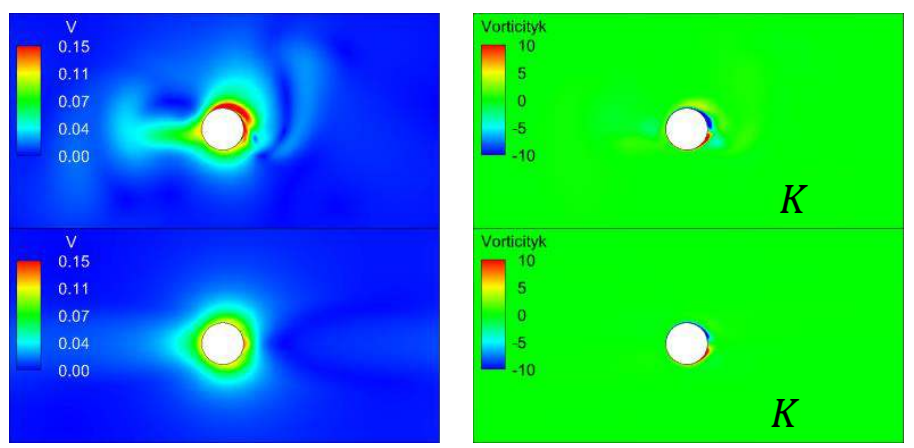

Figure 10: Velocity (left) and vorticity (right) fields for $\beta=\mathbf{5 2 6 0}$, $\mathrm{K}_{\mathrm{C}}=\mathbf{4}$
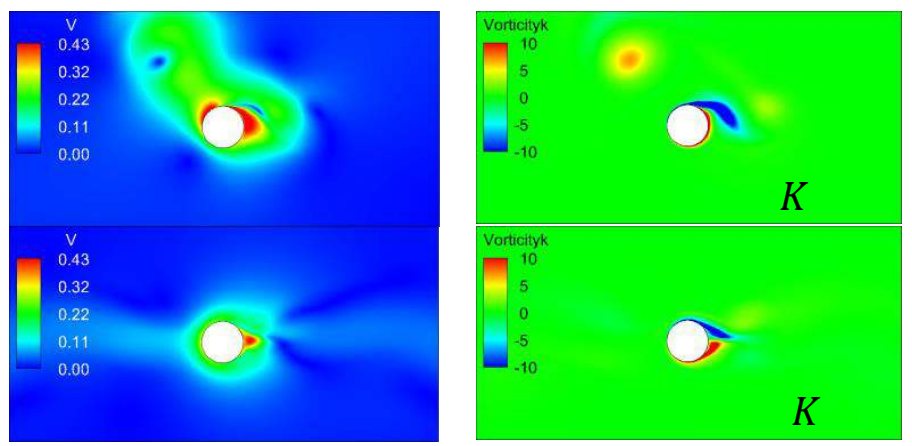

Figure 11: Velocity (left) and vorticity (right) fields for $\beta=\mathbf{5 2 6 0}$, $K_{C}=10$ 

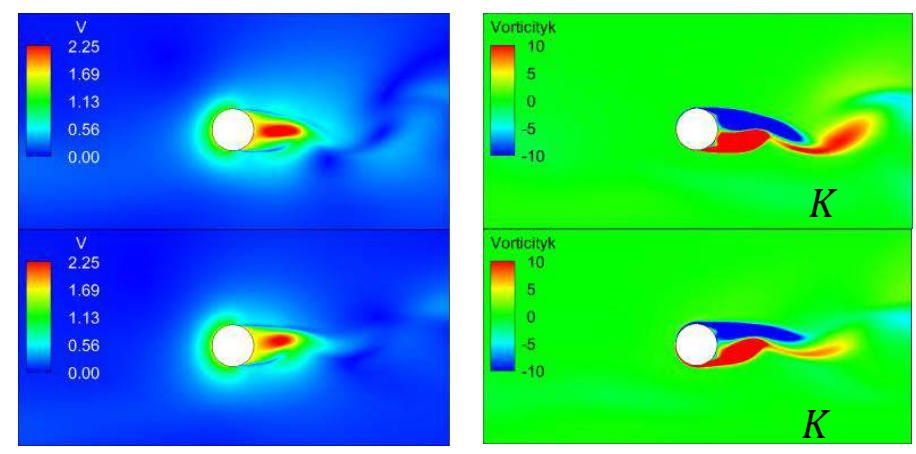

Figure 12: Velocity (left) and vorticity (right) fields for $\beta=\mathbf{5 2 6 0}$, $\mathbf{K}_{\mathrm{C}}=\mathbf{5 0}$

\section{Validation results}

The results obtained on the circular section are presented in Figure 13 and Figure 14 for the solvers ISIS-CFD and Star$\mathrm{CCM}+$ with the turbulence model SST $K-\omega$.

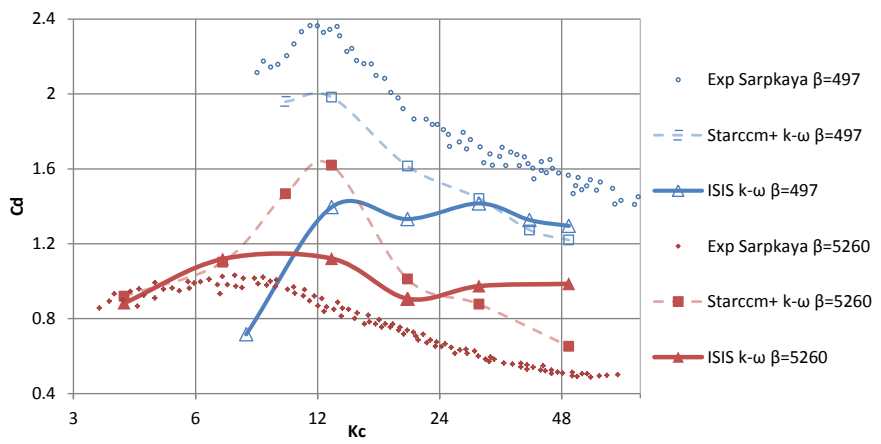

Figure 13: Comparison of ISIS and Star-CCM+ to experimental drag coefficient in oscillatory flow on circular sections

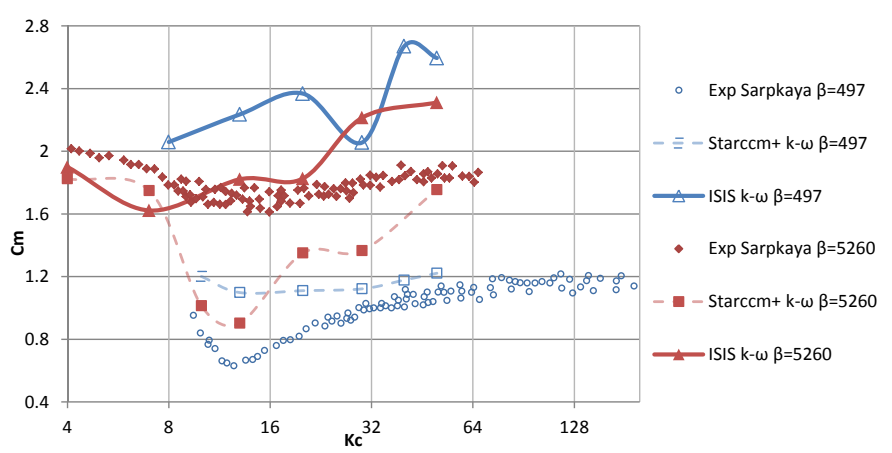

Figure 14: Comparison of ISIS and Star-CCM+ to experimental added mass coefficient in oscillatory flow on circular sections

The results show that both CFD solvers ISIS and Star$\mathrm{CCM}+$ provide results of the same general tendency for $\mathrm{C}_{\mathrm{d}}$, except for some particular points. The added mass coefficient is well estimated by ISIS for the highest Stokes parameter, but the results show significant differences for the lowest Stokes parameter. These results show the difficulty to solve this particular problem, even after completing a sensitivity study on the mesh, on timesteps and on the turbulence model. Regarding the low $\mathrm{Kc}$ for which these tests are performed, laminar computations should be performed as well in the future.

\section{SHIP SECTIONS IN FORCED OSCILLATION MOTIONS}

\section{Experimental set up}

These results are issued from Vugts' experiments (1968). For various cross sections, the hydrodynamic coefficients of $2 \mathrm{D}$ cylinders are determined by forced oscillation tests. In this paper, results are presented on a square section with rounded corners and on a rounded section (Figure 15 and Figure 16). The experiments are performed in the main basin of the Delft shipbuilding laboratory, which dimensions are $142 \mathrm{~m}$ long and $4.20 \mathrm{~m}$ width. Since the draughts of cylinders vary, the water depth varies between $1.80 \mathrm{~m}$ and $2.25 \mathrm{~m}$. The motion of rotation is around point $\mathrm{G}$, and to study various draughts, the water level decreases while the model remains at the same location. The equations of motions are written around point $\mathrm{O}$. The damping coefficients $b$ are followed by indices referring to the imposed motion or coupled degrees of freedom. They are expressed under a non-dimensional expression, depending on the forced motion amplitude $A$ and the breadth $B$ of the section.

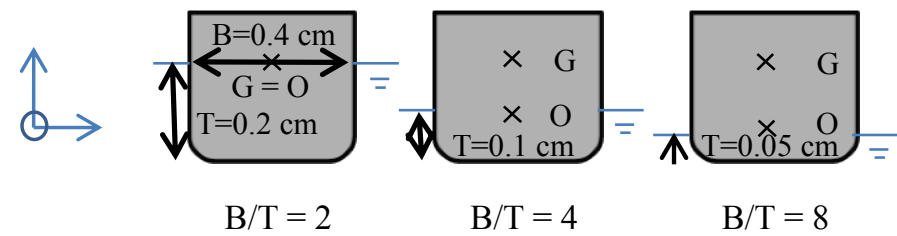

Figure 15: Sketch of the square ship sections in experiments from Vugts (1968)

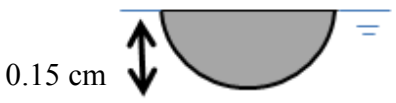

Figure 16: Sketch of the rounded ship section in experiments from Vugts (1968)

\section{Numerical model}

The numerical model is performed as a $2 \mathrm{D}$ case with ICARE and Star-CCM+. With Star-CCM+, a deforming mesh technic is used with a VOF free surface treatment. A sketch of the various mesh zones is presented in Figure 17. The inner zone moves with the forced motion, a transition zone can be deformed and an outer zone is fixed. With ICARE, the free surface tracking method enables to deform the mesh of the free surface (Figure 18). 


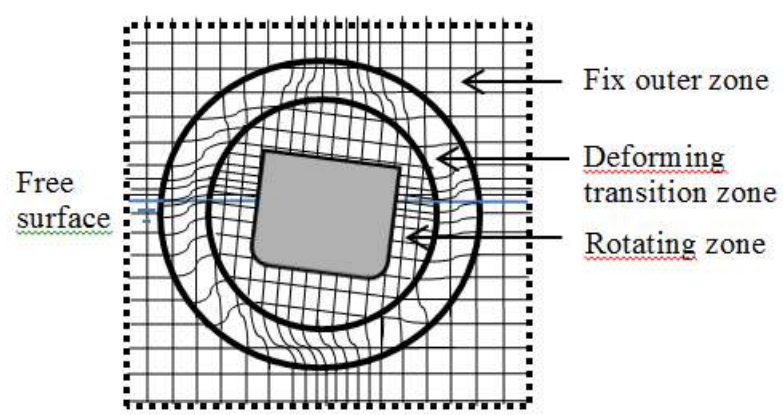

Figure 17: Sketch of the deforming mesh for free surface computations with VOF free surface treatment in Star-CCM+

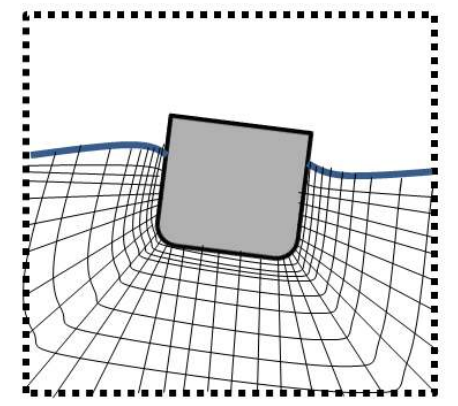

Figure 18: Sketch of the mesh for free surface computations with tracking method in ICARE

\section{Timestep and mesh convergence}

In the comparison to the Vugts results, four mesh densities are tested and five timesteps. The convergence is performed for forced oscillations in heave, for a period of $1.4 \mathrm{~s}$ and for the sections with the ratio Breadth/Draught $=\mathrm{B} / \mathrm{T}=2$. The timestep is considered to be converged with 200 timesteps per period. The mesh 2 is retained to perform the validation study.

\begin{tabular}{|c|c|c|c|c|}
\hline & mesh1 & mesh2 & mesh3 & mesh4 \\
\hline$\Delta t / T \quad 25$ & $76 \%$ & $75 \%$ & $82 \%$ & - \\
\hline$\Delta t / T \quad 50$ & $36 \%$ & $35 \%$ & $36 \%$ & $50 \%$ \\
\hline$\Delta t / T \quad 100$ & $16 \%$ & $14 \%$ & $15 \%$ & $19 \%$ \\
\hline$\Delta t / T \quad 200$ & $9 \%$ & $5 \%$ & $7 \%$ & $5 \%$ \\
\hline$\Delta t / T \quad 400$ & $6 \%$ & $2 \%$ & $3 \%$ & $1 \%$ \\
\hline$\Delta t / T \quad 800$ & $5 \%$ & $2 \%$ & $4 \%$ & REF. \\
\hline
\end{tabular}

Figure 19: Errors in the timestep and mesh convergence study compared to a reference (Mesh 4 and 800 timesteps per period)

for the square section, rounded corners and free surface

\section{Validation results}

Damping coefficients of various ship sections (Vugts, 1968) are calculated with ICARE. The results are in good agreement with the experiments in heave and sway but results show significant differences in roll motions. Roll damping coefficients are then calculated with Star-CCM+ and show a remarkably good agreement with ICARE results. A study from
Bonfiglio (2012) with OpenFoam solver also shows a comparable tendency of the damping coefficients in roll.

The experimental results should also be considered by taking into account the impression of the author that "it was expected that the measurements when rolling were difficult" and because the rotation point is different from the reduction point $(\overrightarrow{O G} \neq 0)$, "the experimental accuracy may be questionable. Many correction terms appear, which are subject to experimental errors in themselves". However, an experimental study from Rae (2003) reproduces one of the experiments from Vugts on the square section and found similar results in roll.

In Vugts (1968), the author says that "no explanation can be given for the fact that in [Figure 23] a roll amplitude of 0.05 gives higher experimental values than for an amplitude of 0.1 ". In this case, CFD results provide better results. The experimental uncertainties on roll have a direct impact on coupled damping coefficients. Figure 25 shows that CFD is able to obtain these coupled coefficients and provide converged results between the two solvers. An additional rounded section is studied with ICARE in heave, sway and roll. Figure 26 to Figure 28 show that results are in good agreement with experiments.

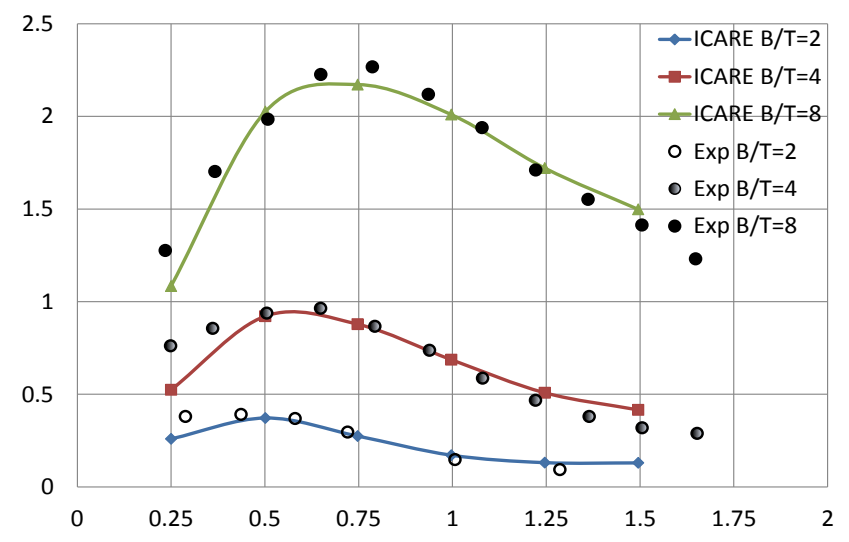

Figure 20: Square ship section in heave

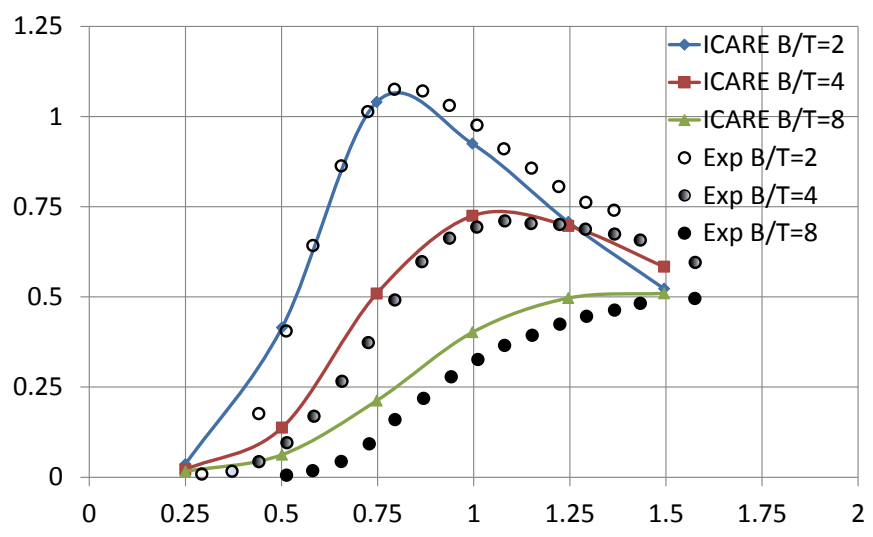

Figure 21: Square ship section in sway 


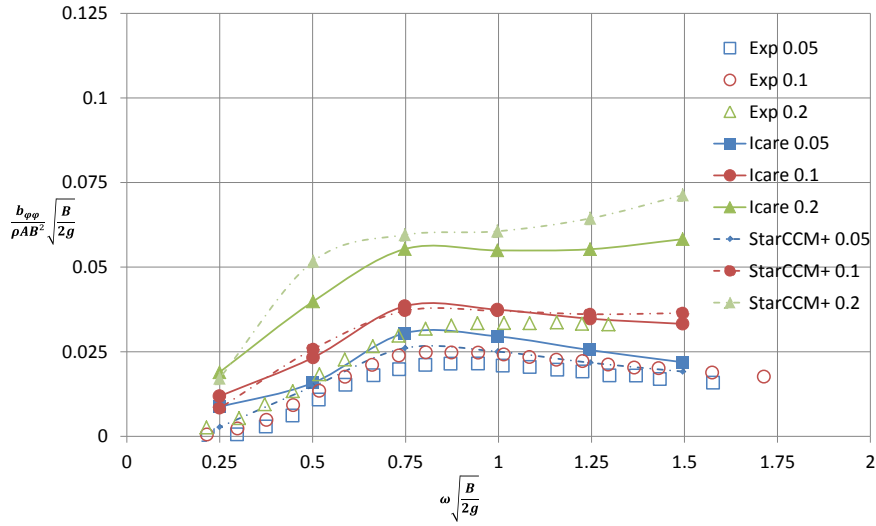

Figure 22: Damping coefficient in Roll for $B / T=2$

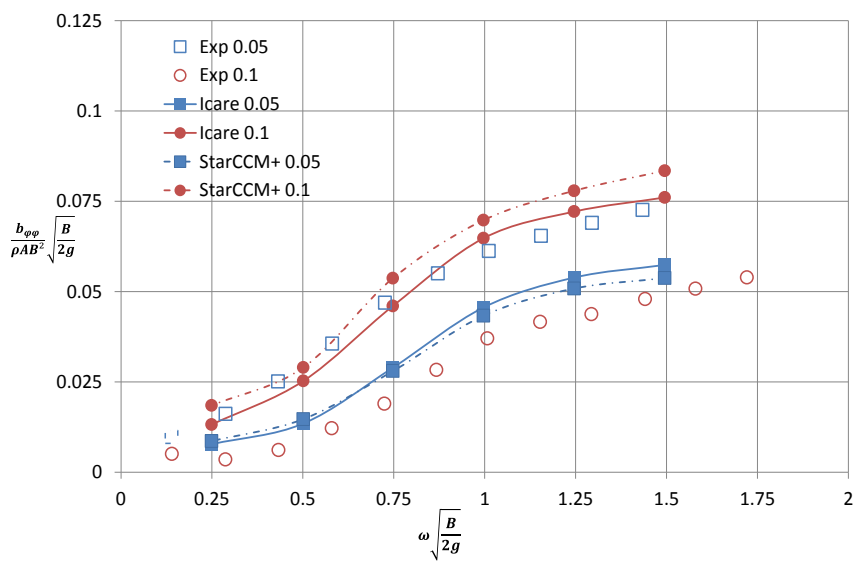

Figure 23: Damping coefficient in Roll for $B / T=4$

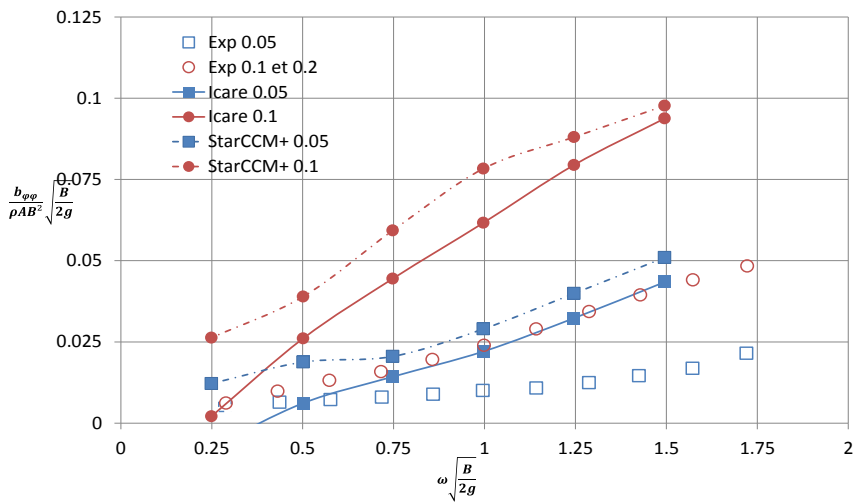

Figure 24: Damping coefficient in Roll for $B / T=8$

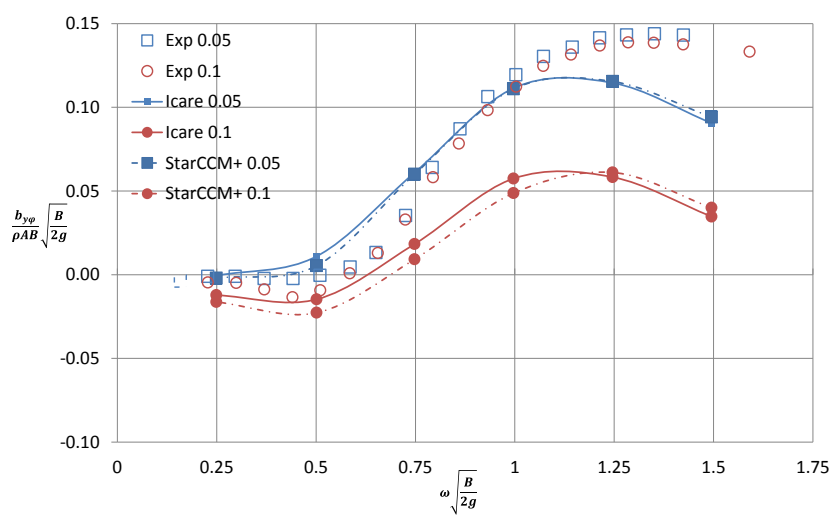

Figure 25: Coupled damping coefficient in Roll-Sway for $B / T=8$

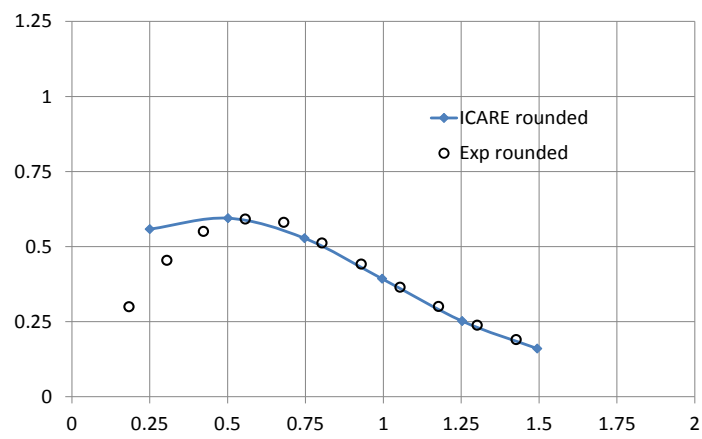

Figure 26: Rounded ship section in heave

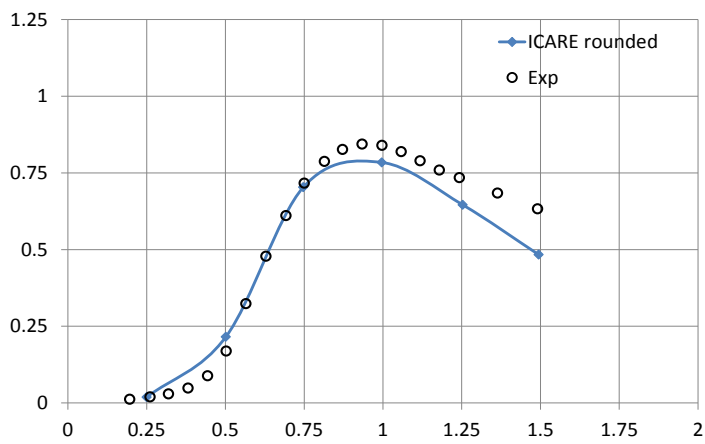

Figure 27: Rounded ship section in sway

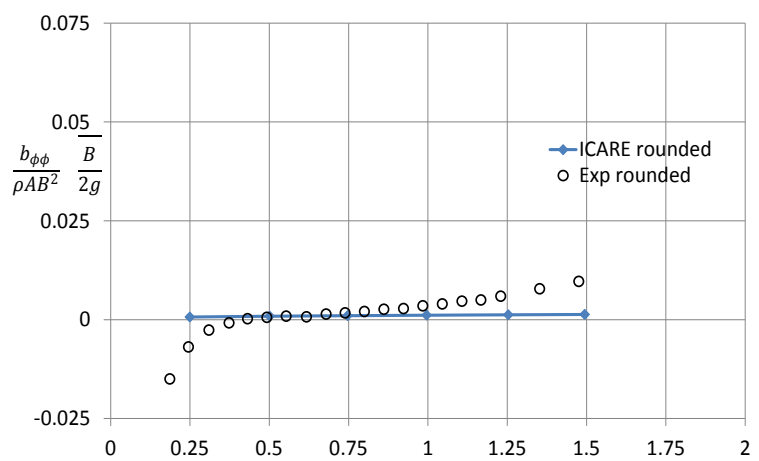

Figure 28: Rounded ship section in roll 


\section{CONCLUSION}

Various numerical parameters were studied in order to propose a methodology insuring the accuracy of CFD computations. The mesh convergence study enabled to determine that a mesh refinement of around 2 to $2.5 \%$ of the body characteristic length is sufficient in the wake area. It is recommended to apply this refinement parameter over the length of the wake area where the body passes, since the body passes through its own wake in forced oscillation motions. The total number of cells can be reduced by mainly refining locations where specific flow detachment occurs, such as body corners or sharp edges. A timestep convergence study has shown that accurate results are obtained from 200 timesteps per period. A direct resolution of the boundary layer is recommended by using a $y+$ equal or lower than one.

Three validation test cases have been performed using these numerical parameters. On the circular section, comparison to Sarpkaya tank test results show that numerical results are dependent on the chosen turbulence model. Because of the absence of sharp edges, the flow detachment is not imposed at one precise location, which is difficult to solve by two-equation turbulence models. Indeed, this problem is one of the most difficult ones to solve in CFD.

On the square section of Bearman, the CFD results are in good agreement with experiments at high $\mathrm{Kc}$ and show more discrepancies at low $\mathrm{Kc}$ for which experiments were more difficult to perform and for which laminar computations could be more adapted. The ship sections from Vugts are very well approximated by CFD in heave and sway. However, the roll damping coefficients are very different in CFD compared to experiments. In this case, Vugts himself mentioned several warnings about the accuracy of experiments. A good indicator for CFD results accuracy is that very similar results are obtained with ICARE and Star-CCM+. Bonfiglio (2012) with OpenFoam solver also shows a similar tendency of the damping coefficients in roll. This fact improves the confidence one could have in these CFD results in roll.

\section{ACKNOWLEDGMENTS}

The author acknowledges the support of the French Agence Nationale de la Recherche (ANR) under reference ANR-2011MONU-018

\section{REFERENCES}

Alessandrini, B., Delhommeau, G., "Simulation of threedimensional unsteady viscous free surface flow around a ship model", International Journal for Numerical Methods in Fluids, vol 19, 1994, pp. 321-342

Alessandrini, B., Delhommeau, G., "A fully coupled Navier-Stokes solver for calculations of turbulent incompressible free surface flow past a ship hull", International Journal for Numerical Methods in Fluid, vol 29, 1999, pp. 125142

Atluri S., Magee A., Lambrakos, K. "CFD as a design tool for hydrodynamic loading on offshore structures", proceedings of International conference on ocean, Offshore and Arctic engineering, 2009, 2009-79502

Bearman et al, "Forces on cylinders in viscous oscillatory flow at low Keulegan-Carpenters numbers", J. Fluid Mechanics, 1985, vol. 154, pp. 337-356

Bonfiglio L., Brizzolara S., Chryssostomidis C., "Added mass and damping of oscillating bodies: a fully viscous numerical approach", Recent advances in Fluid Mechanics, Heat \& Mass transfer and biology, 2012, p210

Kinnas S., Yu YH, Lee H., Kakar K. "Modelling of oscillating flow past a vertical plate", Proceedings of the $13^{\text {th }}$ International offshore and polar engineering conference, Hawaii, 2003

Rae H. Yuck, Doncg H.Lee, Hang S. Choi, "Estimation of roll damping coefficients for non-conventional mid-ship sections", Proceedings of the $13^{\text {th }}$ International offshore and polar engineering conference, Hawaii, 2003

Sarpkaya, "In-line and transverse forces on cylinders in oscillatory flow at high Reynolds Numbers", Offshore technology conference OTC-2533, 1976

Tao, L., Thiagarajan K., "Low Kc flow regimes of oscillating sharp edges. II. Hydrodynamic forces”, Applied ocean research, 2003, 25 (2), 53-62

Visonneau et al. "Anisotropic grid adaptation for RANS simulation of a fast manoeuvring catamaran", $4^{\text {th }}$ High performance Yacht design conference, Auckland, 2012

Vugts, J.H, "The hydrodynamic coefficients in swaying, heaving and rolling cylinders in a free surface", 1968, Netherlands Ship Research Centre TNO Report No. 112 S, pp. $251-27$ 